\title{
Interfaces entre Modelagem Matemática, Raciocínio e Pensamento Estatístico
}

\author{
Interfaces between Mathematical Modeling, Reasoning and Statistical \\ Thinking
}

Andréa Pavan Perin

Celso Ribeiro Campos

\begin{abstract}
Resumo: A Educação Estatística é uma área de estudos e pesquisas que se formou mediante a busca por compreender os processos de ensino e de aprendizagem de Estatística. No contexto brasileiro, em tais estudos, tem ganhado relevância o desenvolvimento de três competências, a literacia, o raciocínio e o pensamento alicerçados em ambientes de Modelagem Matemática. Esse artigo, de natureza teórica, tem como objetivo traçar um paralelo entre as etapas da Modelagem Matemática, os elementos do pensamento e os tipos de raciocínio estatístico, com a finalidade de apontar o que há de comum nas etapas propostas entre eles e, assim, contribuir com 0 desenvolvimento das pesquisas na área. $O$ estudo apontou que o ambiente de Modelagem Matemática possui relações significativas com os tipos de raciocínio e os elementos do pensamento estatístico, o que nos permite afirmar que esse ambiente pode favorecer 0
\end{abstract} desenvolvimento dessas competências.

Palavras-chave: Educação Estatística. Pensamento Estatístico. Raciocínio Estatístico. Modelagem Matemática.

Abstract: Statistical Education is an area of studies and research that was formed through the search to understand the Statistics' teaching and learning process. In the Brazilian context in such studies, the development of three competencies, literacy, reasoning and thinking, based on Mathematical Modelling environments, has gained relevance. This article, of a theoretical nature, aims to draw a parallel between the stages of Mathematical Modelling, the elements of thought and the types of statistical reasoning, with the purpose of pointing out what is common in the steps proposed between them and thus contributing with the development of research in the area. The study pointed out that the Mathematical Modeling environment has significant relationships with the types of reasoning and the elements of statistical thinking, which

Andréa Pavan Perin Doutora em Educação Matemática. Professora da Faculdade de Tecnologia de Itapetininga (FATEC). São Paulo, Brasil. iD orcid.org/0000-0002-2791-7682 $\triangle$ andreapavanperin@gmail.com

Celso Ribeiro Campos Doutor em Educação Matemática. Professor do Programa de Estudos Pós-Graduados em Educação Matemática da Pontifícia Universidade Católica de São Paulo (PUCSP). São Paulo, Brasil. iD orcid.org/0000-0001-7371-2437 $\triangle$ crcampos@pucsp.br

Recebido em 07/06/2020 Aceito em 04/07/2020 Publicado em 24/07/2020 allows us to affirm that this environment can favor the development of these competences.

Keywords: Statistics Education. Statistical Thinking. Statistical Reasoning. Mathematical Modelling.

\section{Introdução}

A Estatística, por ser uma ciência de análise de dados, está presente em todos os contextos. Por essa razão, compreendê-la é fundamental para que as pessoas possam entender e avaliar dados estatísticos que circulam nos variados meios de comunicação. 
Reconhecida a frequência da Estatística no cotidiano das pessoas, pesquisadores da Educação Estatística começaram a discutir a importância dessa ciência ser ensinada desde a educação básica até o ensino superior. Tal discussão foi pontuada, inicialmente, por meio de dois documentos, sendo um da American Statistical Association (ASA) e outro do National Council of Teachers of Mathematics (NCTM), publicados nos anos de 1960 e 1967, respectivamente. Dada a expressividade adquirida por esses documentos, a partir de 1989, revistas importantes da área publicaram artigos que destacavam duas ações que mereceriam atenção nos anos seguintes: inserir a Estatística a todos os níveis escolares e dar maior atenção às formas de conduzir os processos de ensino e de aprendizagem dessa ciência (ZIEFFLER, GARFIELD e FRY, 2018).

No contexto brasileiro, a preocupação com os processos de ensino e de aprendizagem de Estatística também tem ganhado espaço nos documentos oficiais. No final da década de 1990, com a publicação dos Parâmetros Curriculares Nacionais (PCN), a Estatística e a Probabilidade foram incorporadas oficialmente à estrutura curricular da educação básica (SAMÁ, 2018). Nesse documento, elas aparecem como uma área da Matemática responsável pelo tratamento da informação. Mais recentemente, a promulgação da Base Nacional Comum Curricular (BRASIL, 2018) ratificou a inserção da Estatística e da Probabilidade na educação básica e sugeriu a abordagem de conceitos estatísticos por meio de situações da vida cotidiana, das ciências e da tecnologia (CAZORLA, SILVA e SANTANA, 2018). De modo geral, ampliou-se a quantidade de conteúdos relacionados à unidade temática Estatística e Probabilidade, incluindo conteúdos como gráficos dot-plot e com ênfase em projetos (BRASIL, 2018), sendo este último também conhecido como investigação estatística ou ciclo investigativo.

Ainda no contexto brasileiro, a publicação dos PCN levou muitos pesquisadores a desenvolver estudos voltados à área da Educação Estatística. Tais estudos, em sua maioria, defenderam que o planejamento das atividades deve ser baseado no desenvolvimento de três competências: literacia, raciocínio e pensamento estatísticos, sem as quais não seria possível a aprendizagem de ideias essenciais da Estatística. Além disso, reforçaram que o desenvolvimento dessas competências seria passível de maior sucesso quando os alunos experimentam todas as etapas de uma investigação estatística: formulação de questões; coleta de dados; análise de dados; interpretação e divulgação dos resultados. Essas etapas sugerem que as atividades de Estatística possuem uma relação próxima de um ambiente de aprendizagem denominado Modelagem Matemática na perspectiva da Educação Matemática (CAMPOS, 2007; CAMPOS, WODEWOTZKI e JACOBINI, 2011; MACHADO, 2017; PERIN, 2019; SOARES, 2017; 
TRAINOTTI, 2019).

As pesquisas mencionadas abordam os trabalhos de Modelagem Matemática em sala de aula nos quais os alunos, reunidos em grupos, escolhem temas, coletam dados, os simplificam e organizam, tendo o professor como co-participante em todas os momentos mencionados. Ao reportarem ao ambiente de Modelagem Matemática nas pesquisas em Educação Estatística, busca-se as implicações, contribuições e/ou possiblidades metodológicas que esse ambiente pode trazer trazer para o ensino e a aprendizagem de conteúdos de Estatística, bem como compreender aspectos envolvidos num processo de investigação estatística. Entendemos que o trabalho com os conteúdos de Estatística devem priorizar o desenvolvimento de tipos específicos de raciocínio estatístico, além de um entedimento de como, quando e por que as ferramentas estatíscas devem ser utilizadas. Desse modo, este artigo tem como objetivo traçar um paralelo entre as etapas da Modelagem Matemática, os elementos do pensamento estatístico e os tipos de raciocínio estatístico, com a finalidade de apontar o que há de comum nas etapas propostas entre eles e assim contribuir com o desenvolvimento das pesquisas na área. 0 estudo apresentado tem origem nos resultados parciais da pesquisa de doutorado da primeira autora do artigo, a qual foi feita mediante uma confrontação dos resultados da Modelagem Matemática com os preceitos da Educação Estatística e da Educação Crítica. Nesse trabalho, observamos algumas convergências que ora pretendemos aprofundar, de modo a responder a seguinte questão: Quais elementos do pensamento e do raciocínio estatístico podem ser explorados mais intensamente em cada uma das etapas da Modelagem Matemática?

Trabalhamos com a hipótese de que há diversas inferfaces que incluem as etapas de modelagem e os diversos tipos e níveis de raciocínio e de pensamento, as quais buscamos expor nas próximas seções. Assim, na sequência, apresentamos a nossa metodologia e a fundamentação teórica sobre Modelagem Matemática, raciocínio e pensamento estatísticos, a fim de destacar as evidências que nos conduzem ao objetivo desta pesquisa.

\section{Metodologia}

De acordo com Almouloud e Silva (2019), pesquisar significa procurar respostas para certas indagações, de forma planejada, utilizando procedimentos sistemáticos, baseados no raciocínio lógico, no intuito de encontrar soluções para os problemas propostos mediante 0 emprego de métodos científicos. Ainda segundo os autores, o processo de pesquisa é um conjunto 
de operações sucessivas realizadas para coletar sistematicamente informações válidas sobre um fenômeno observável para explicá-lo ou compreendê-lo. Dessa forma, os procedimentos metodológicos têm por função a sistematização das etapas realizadas em um determinado estudo para assegurar a sua confiabilidade e validade. Nesse sentido, os procedimentos devem ser concebidos para construir os meios que permitem alcançar o objetivo geral da pesquisa.

Adicionalmente, assim como Coutinho e Campos (2019), entendemos a metodologia como sendo a linguagem estruturante do pensamento acadêmico/científico, ou seja, "aquilo que organiza o raciocínio lógico/analítico/cognitivo pertinente a uma argumentação formal cuja finalidade é a validação ou refutação de uma ou mais hipóteses" (p. 83).

Segundo Fiorentini e Lorenzato (2006), partindo de um problema ou da questão de investigação, existem dois modos de chegar às conclusões, quais sejam: por meio de uma pesquisa de campo ou de laboratório, a qual conduz à coleta e análise de dados/material empírico ou experimental; e por meio da pesquisa teórica ou bibliográfica, que surge com base na literatura, pesquisas ou estudos precedentes, na qual é preciso coletar/organizar dados ou documentos para um tratamento analítico de informações. A questão de pesquisa que ora buscamos responder se encaixa nesse segundo tipo descrito pelos autores. Complementarmente, dada a questão de investigação e os objetivos desta pesquisa, podemos dizer que é uma pesquisa de natureza qualitativa, pois o que se busca é o entendimento de ligações entre os elementos da Educação Estatística e da Modelagem Matemática. Sendo assim, a busca está direcionada à compreensão da manifestação desses elementos, e não ao tratamento quantitativo de dados.

Iniciamos nossa explanação sobre a importância do tema deste estudo citando a evolução que se sucedeu à publicação dos documentos da ASA e do NCTM. Citamos também alguns documentos brasileiros que reforçaram a importância do ensino de Estatística na educação básica, quais sejam os PCN e a BNCC. Tendo como ponto de partida esses documentos citados, vamos trilhar nosso caminho em busca da resposta à nossa questão de pesquisa por meio do nosso quadro teórico. Almouloud e Silva (2019) pontuam que o referencial teórico é a base que sustenta qualquer pesquisa científica, "esse referencial implica na perspectiva em que um problema será abordado, as diretrizes para sua elaboração e avaliação de sua relevância a priori e a posteriori" (p. 49). Ainda segundo os autores, o referencial teórico pode garantir a sistematização do conhecimento, distinguindo esse conhecimento do senso comum com base nos procedimentos usados na pesquisa. 
O referencial teórico que nos permitirá alcançar o objetivo desta pesquisa começa com a descrição das competências de raciocínio e pensamento estatísticos. O raciocínio estatístico será analisado com base nos estudos de Pfannkuch (2018) e Biehler et al. (2018), entre outros. Já sobre o pensamento estatístico, nos basearemos principalmente nos estudos de Snee (1990, 1999) e Wild e Pfannkuch (1999). Nossa ideia é entender os elementos que compõem essas competências, suas características e padrões de desenvolvimento. Feito isso, iremos estudar a Modelagem Matemática segundo a percepção de Burak (1992, 2004 e 2019), pontuando as características das diversas fases que compõem essa estratégia pedagógica.

Por fim, com base nesse referencial teórico, buscaremos apontar as características comuns que puderam ser observadas, pontuando as interfaces que dão suporte aos objetivos desta pesquisa.

\section{0 raciocínio estatístico}

O raciocínio estatístico define o modo com que os indivíduos raciocinam com as ideias estatísticas e dão sentido à informação estatística. Tem subjacente a compreensão conceitual e a conexão de importantes ideias, como variação, distribuição, centro, dispersão, associação e amostragem ou a combinação de ideias sobre dados e incerteza que conduzem à realização de inferência (PFANNKUCH, 2018).

Para que os alunos possam desenvolver essa competência, as tarefas em sala de aula devem ser preparadas com o objetivo de levá-los ao desenvolvimento de ideias estatísticas centrais. É necessário que elas levem ao aprofundamento da compreensão conceitual em Estatística, ao invés da aprendizagem de procedimentos e representações específicas. Algumas dessas ideias centrais, segundo Biehler et al. (2018), são:

- Dados: o objetivo é perceber a necessidade dos dados para tirar conclusões e fazer avaliações. Aspectos ligados à obtenção dos dados que sejam válidos devem ser também contemplados.

- Distribuição: a ideia é levar os alunos a ter uma visão global dos dados de modo a desenvolverem a noção de distribuição.

- Variabilidade e centro: deve-se incentivar a conjugação da análise de medidas de tendência central com a dispersão dos dados, medida, por exemplo, pela amplitude da 
amostra e pela amplitude interquartis.

- Amostragem, inferência e probabilidade: saber como as amostras estão relacionadas com a população e o que pode ser inferido com base em uma amostra, levando os alunos a compreender que as decisões se baseiam em amostras.

Essa competência, portanto, valoriza a análise dos dados, a capacidade de raciocinar sobre eles e usá-los de modo efetivo e crítico na tomada de decisões. Dessa forma, ela contrasta com uso de fórmulas que requerem cálculos morosos, repetitivos e sem significado para os alunos.

O raciocínio estatístico concerne ao trabalho com as ferramentas estatísticas, não direcionado somente a operar com elas, mas atento aos seus significados mais profundos. Tratase da capacidade de interligar as ideias associadas a ferramentas estatísticas, de tomar ciência do seu sentido, da mensagem subjacente e, principalmente no contexto em que são utilizadas.

Em estudo anterior (PERIN, 2019), consideramos que o raciocínio estatístico abarca a compreensão de como se conduzem e se desenvolvem investigações estatísticas, com ideias claras da natureza e dos processos envolvidos. Inclui a capacidade de utilizar os procedimentos estatísticos adequados a cada uma das etapas, levando em consideração as particularidades do contexto. O raciocínio engloba também o entendimento das ligações com as ideias matemáticas presentes nos procedimentos estatísticos, como, por exemplo, explicar as relações entre o nível de confiança e o tamanho do intervalo de confiança. Essa competência compreende, ademais, a capacidade de se comunicar estatisticamente, isto é, explicar procedimentos e ideias estatísticas.

De forma a proporcionar o desenvolvimento dessa capacidade, Ben-Zvi e Aridor-Berger (2016) sugerem uma abordagem curricular que enfatize e promova a compreensão da Estatística como um processo investigativo para resolver problemas reais em que os alunos se sintam motivados para: formular suas próprias questões, selecionar e aplicar métodos (gráficos e numéricos) apropriados para analisar dados; desenvolver e avaliar inferências e previsões baseadas em dados e relacionar a análise com a questão original em estudo.

\section{0 pensamento estatístico}

O pensamento estatístico compreende estratégias mentais associadas à tomada de decisão em todas as etapas do ciclo investigativo. Inclui um entendimento de como os modelos são usados para simular fenômenos, de como os dados são produzidos para estimar a 
probabilidade e como, quando e por que as ferramentas de inferência existentes podem ser usadas para auxiliar um processo investigativo. Snee (1990, p. 118), define o pensamento estatístico como um

processo de raciocínio que reconhece que a variação está em tudo ao nosso redor e presente em tudo que fazemos, que todo trabalho é uma série de processos interligados; e que identificar, caracterizar, quantificar, controlar e reduzir a variação fornece oportunidades de melhoria".

Nesse sentido, podemos associar o pensamento estatístico à ideia de variação, mais especificamente à produção e análise de dados e à tomada de decisão.

Wild e Pfannkuch (1999), após realizarem estudos sobre o pensamento estatístico entrevistando profissionais da área, professores e alunos de Estatística, concluíram que essa competência está baseada em quatro dimensões: ciclo investigativo, ciclo interrogativo, tipos de pensamento e disposições.

O ciclo investigativo refere-se à forma de agir e pensar durante uma investigação estatística, sido adaptado para PPDAC (problem, plan, data, analysis, conclusions) 1 . Ainda segundo os autores, esse ciclo objetiva resolver problemas reais, geralmente tencionando mudar um sistema para melhorá-lo. A qualidade desse ciclo vai depender também do conhecimento do contexto. Vale destacar que tais considerações vão ao encontro das assinaladas por Snee (1990).

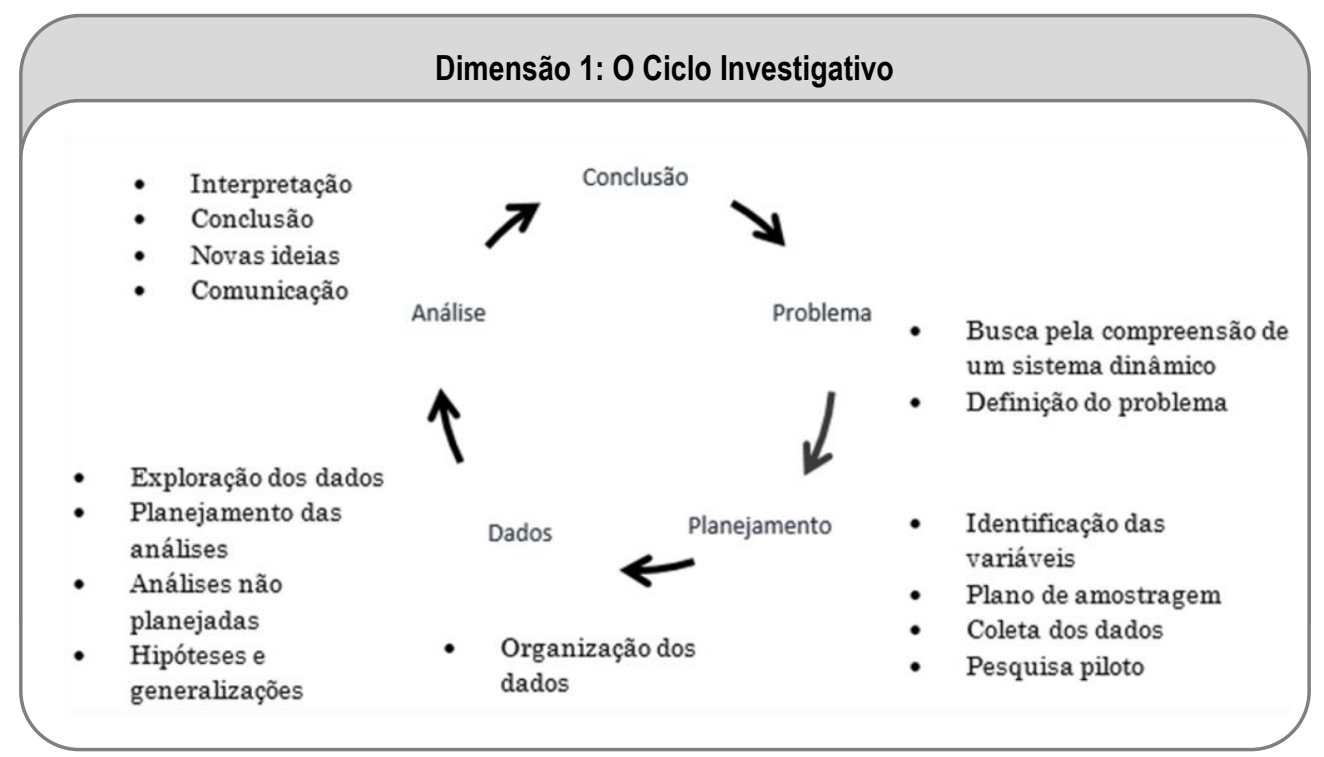

Figura 1: Dimensões do ciclo investigativo e atividades envolvidas (Adaptado de Wild e Pfannkuch, 1999, p. 226)

\footnotetext{
1 Traduzido por nós como: Problema, planejamento, coleta de dados, análises e conclusões.
} 
O ciclo interrogativo é um processo genérico do pensamento e constante durante a resolução de um problema estatístico. Ele relaciona-se ao levantamento de questões nos níveis macro e micro da pesquisa. Este ciclo engloba cinco atividades: geração de ideias - compreende geração de ideias e possibilidades, que pode ocorrer em grupo ou individualmente, pode ser oriunda do contexto ou do conhecimento estatístico e aplicada à pesquisa corrente ou indicações para futuras pesquisas; busca de informações - consiste na busca de informações para dar continuidade à pesquisa, e pode ocorrer em fontes internas (o que já conhecia) ou externas (literatura ou outras pessoas); interpretar - é o processo que envolve os atos de ler, ver, ouvir, traduzir, resumir internamente, comparar e conectar; criticar - envolve 0 ato de checar os pontos a favor e contra o resultados que se têm em mãos e, como citado, essa atividade relaciona-se a fontes internas e externas; julgar - envolve o questionamento sobre a confiabilidade das informações, utilidade das ideias, praticidade dos planos, conformidade com o contexto e entendimento estatístico, necessidade de outras pesquisas etc.

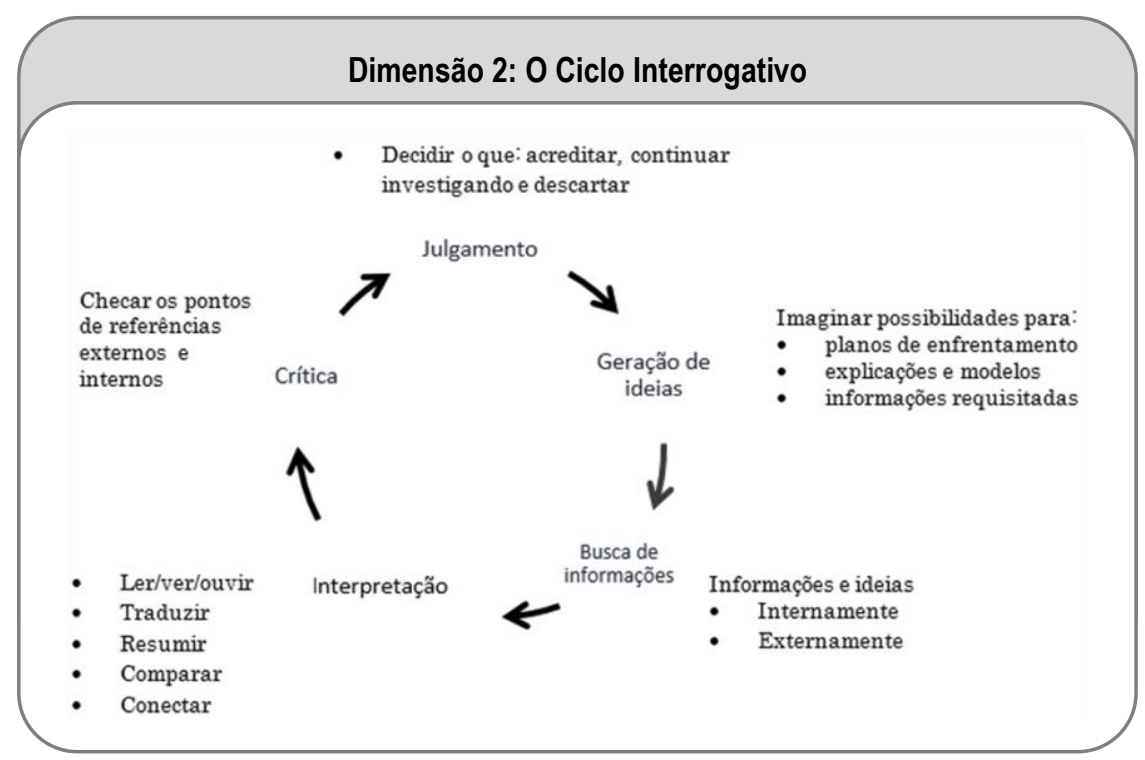

Figura 2: Dimensões do ciclo interrogativo e atividades envolvidas (Adaptado de Wild e Pfannkuch, 1999, p. 226)

A terceira dimensão, denominada tipos de pensamento, é composta por dois tipos, o pensamento geral e o pensamento fundamental, e cada um possui seus elementos, conforme mostrado na Figura 3. 


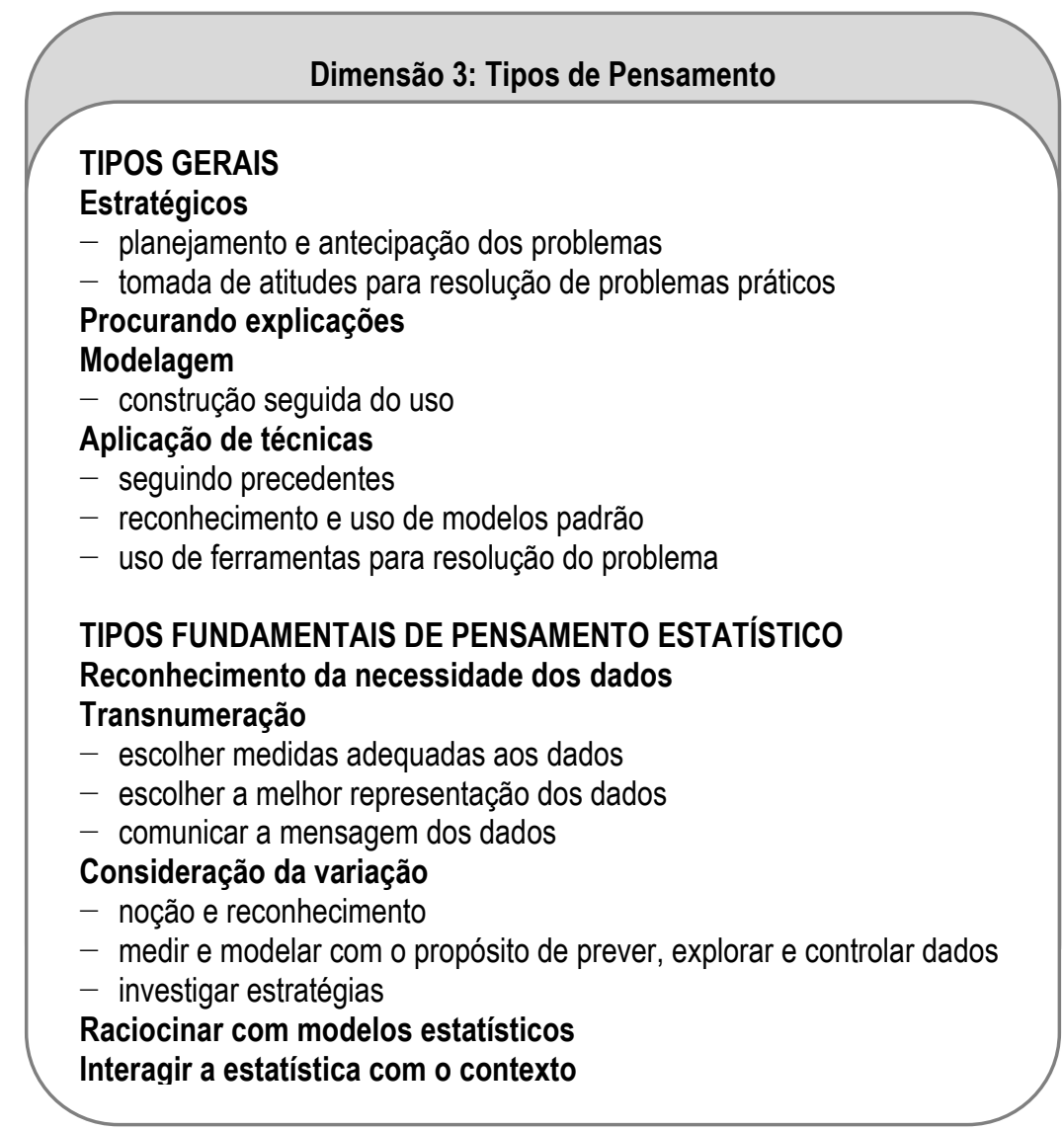

Figura 3: Tipos de pensamento e respectivas atividades envolvidas (Adaptado de Wild e Pfannkuch, 1999, p. 226)

O pensamento geral refere-se ao planejamento do ciclo investigativo, visando a algumas estratégias que conduzem à investigação e, por meio dele, elaboram-se questões do tipo: 0 que faremos? Como faremos? Temos dinheiro e material suficiente? Quais são os prazos? Os dados podem apresentar limitações? Tais questionamentos levam ao planejamento de como as tarefas serão realizadas, divisão de cada tarefa de pesquisa em subtarefas, definição de prazos para a realização das tarefas, divisão do trabalho no grupo, antecipação de problemas e planejamento para evitá-los etc.

Já o pensamento fundamental, composto de cinco elementos, é caracterizado pela adequação do uso das ferramentas estatísticas em cada uma das etapas da investigação. $\mathrm{Na}$ sequência descrevemos as características de cada um desses elementos.

- Reconhecimento da necessidade dos dados: relaciona-se ao reconhecimento da insuficiência das experiências pessoais para a tomada de decisão, o que conduz à uma deliberada coleta e tratamento de dados para conhecimento/entendimento de um determinado contexto. Pfannkuch e Wild (2003) argumentam que os alunos precisam 
ter consciência de que as conclusões sobre um determinado contexto só podem ser tiradas com base em uma análise cuidadosa de informações pertinentes a ele.

- Transnumeração: ocorre quando encontramos formas de obtenção de dados, por meio de medidas ou classificações, que capturem um elemento significativo da população na qual os dados foram coletados e permeia toda a análise estatística. Pfannkuch e Rubick (2002) acrescentam que a transnumeração ocorre em três estágios. Primeiramente, quando enumeramos ou classificamos medidas relevantes de serem capturadas do mundo real com a intenção de resolver o problema proposto. Em segundo lugar, quando utilizamos as múltiplas representações da Estatística para representar os dados na tentativa de entender o que eles nos podem informar sobre o contexto no qual foram capturados. E finalmente, em terceiro lugar, quando usamos os resumos estatísticos para informar ao público-alvo o que os dados podem dizer sobre o problema inicial.

- Variação: consideração da variação na tomada de decisão em situações de incerteza, com consciência sobre algumas de suas características como: a onipresença da variação do processo, a necessidade dos dados relativos ao processo, a produção dos dados baseados na variação existente, a quantificação da variação e a explanação da variação. Consiste também em ter consciência de que há variações provocadas por causas especiais, como aquelas características que são incomuns às apresentadas por uma amostra, e as de causas comuns que representam as variações naturais esperadas em um processo. Sobre esses aspectos da variação, Snee (1999) acrescenta que devemos ter em mente que toda investigação estatística deve levar em conta dois princípios: controle e aleatoriedade.

- Uso de modelos estatísticos: refere-se ao uso de diferentes tipos de tabelas e gráficos, medidas de centro e dispersão, na tentativa de construir representação e significado para os dados.

- Conhecimento do contexto, conhecimento estatístico e síntese: é ter clareza de que a qualidade de toda investigação estatística depende de um interrelaciomento desses diferentes tipos de conhecimento.

Finalmente, a quarta dimensão é a disposição, a qual está relacionada a categorias de qualidades pessoais, como curiosidade e questionamento, engajamento, imaginação, ceticismo, raciocínio lógico e disposição para aprender. 


\section{Dimensão 4: Disposições}

\section{Ceticismo}

Imaginação

Curiosidade e Atenção

- atento e observando tudo

Abertura

- de ideias e preconceitos

Propensão para procurar significado mais profundo

- sendo lógico

- estando envolvido

Figura 4: Atitudes que devem permear uma investigação estatística (Adaptado de Wild e Pfannkuch, 1999, p. 226)

Refletir sobre as quatro dimensões do pensamento estatístico e os elementos de cada dimensão nos permite entendê-los, bem como as estratégias mentais utilizadas por um indivíduo durante a realização de uma pesquisa empírica. Quando o pensamento estatístico é valorizado, as interpretações prevalecem sobre os cálculos e os conceitos são sempre trabalhados no sentido do porquê fazer. O como fazer decorre da necessidade de precisar fazer.

Assim, para que essas competências possam ser melhor desenvolvidas, o professor deve criar um ambiente no qual os alunos se insiram em uma prática investigativa, em que: trabalhem com temas de seu interesse; estejam em contato com dados que tenham relevância para um determinado contexto; trabalhem com diversidade de variáveis; tenham vivência com a geração e análise dos dados; trabalhem em grupos de forma a favorecer a troca de significado entre os pares; usem a tecnologia de forma a favorecer o entendimento de conceitos; sejam avaliados constantemente pelas relações e julgamentos que estabelecem para um conjunto de dados e não apenas pela aplicação de fórmulas.

Tais ações deslocam-se da ênfase tradicional nas fórmulas, cálculos e procedimentos para um processo de investigação que articula temas de interesse dos alunos mobilizando diferentes saberes. Segundo Eichler (2011) e Moore (2001), esse ambiente é proporcionado quando trabalhamos com projetos e, segundo Pratt e Kazak (2018), os trabalhos desenvolvidos na perspectiva da Modelagem Matemática valorizam essas ações. No contexto brasileiro, autores como, Campos, Wodewotzki e Jacobini (2011), Perin (2019), Evangelista, Evangelista e Santos (2020), também sugerem o trabalho com projetos de Modelagem Matemática para 0 desenvolvimento de competências estatísticas. 


\section{A Modelagem Matemática}

A Modelagem Matemática desenvolvida com base na Educação Matemática tem sido realizada no Brasil desde a década de 1970. Diferentes concepções foram apresentadas no decorrer desse período e estão fortemente relacionadas com o objetivo que se pretende enfatizar ao se desenvolver a Modelagem Matemática no ambiente educacional. De forma geral, busca-se favorecer um ensino de Matemática partindo da necessidade dos envolvidos, de seus interesses, em que os alunos constroem seus conhecimentos (BURAK, 2019).

Para D’Ambrósio (1986), a Modelagem Matemática é um processo muito rico para a sala de aula de Matemática e culmina com a solução efetiva de um problema real e não com a simples resolução formal de um problema artificial. Sendo assim, a prática da Modelagem Matemática busca incentivar os alunos na pesquisa e na resolução de situações problemas do seu cotidiano.

Aproximando da visão desse autor, Barbosa (2001) defende que a Modelagem é um ambiente de aprendizagem no qual os alunos são convidados a indagar e/ou investigar, por meio da Matemática, situações oriundas de outras áreas da realidade, cujo objetivo é alcançar um olhar crítico para a Educação Matemática. Valoriza mais as interações durante o processo de modelagem do que os resultados. Nessa mesma direção, Meyer, Caldeira e Malheiros (2011) defendem a vinculação entre a Modelagem e uma intenção de educar matematicamente, pois entendem a Matemática como um conjunto de regras e convenções que são estabelecidas dentro de determinado contexto social, histórico e cultural, permeadas pelas relações de poder, diferentemente daquela ciência vista como uma simples descoberta. Os referidos autores almejam, por meio dela, ensinar Matemática de modo que os alunos, como agentes ativos, criem mecanismos de reflexão e ação, buscando desenvolver o senso crítico, a capacidade de resolver situações problemas do cotidiano.

Já para Bassanezzi (2011) e Biembengut e Hein (2013), o objetivo principal é criar condições para que o aluno desenvolva a criatividade matemática de forma que aprendam a fazer modelos matemáticos, aprimorando seus conhecimentos. Para esses autores, a construção dos modelos é primordial para que se caracterize o desenvolvimento de atividade de Modelagem Matemática.

Burak (1992) a compreende como uma metodologia de ensino e aprendizagem, a qual possui dois princípios fundamentais: 1) o interesse do grupo; 2) a obtenção e informação de dados do ambiente onde se encontra o interesse do grupo. Dessa forma, "a Modelagem Matemática 
constitui-se em um conjunto de procedimentos, cujo objetivo é estabelecer um paralelo para tentar explicar matematicamente os fenômenos presentes no cotidiano do ser humano, ajudando-o a fazer predições e a tomar decisões" (BURAK, 1992, p. 62).

A Modelagem, como uma estratégia para o ensino de Matemática, tem os encaminhamentos para as atividades sugeridos com base em cinco etapas, conforme Burak (2004): 1) escolha do tema; 2) pesquisa exploratória; 3) levantamento do (s) problema(s); 4) solução do(s) problema(s) e o desenvolvimento de conteúdos e da matemática relacionada ao tema; 5) análise crítica das soluções.

Santos (2015), ao realizar um estado da arte sobre as pesquisas em Educação Estatística, identificou que a Modelagem Matemática no ensino e na aprendizagem de Estatística tem se destacado como elaboração de estratégias didáticas e propostas metodológicas nos variados níveis de ensino.

Tendo em vista a notoriedade que o ambiente de Modelagem Matemática tem alcançado nas pesquisas em Educação Estatística, o que faremos neste artigo será traçar um paralelo entre as etapas da Modelagem Matemática descritas por Burak (2019), os elementos do pensamento estatístico e os tipos de raciocínio, a fim apontar o que há de comum nas etapas propostas entre eles.

\section{Interfaces entre as etapas da Modelagem Matemática, pensamento e raciocínio estatístico}

1) Escolha do tema - O processo de Modelagem Matemática parte de temas propostos pelo grupo, constituído por três ou quatro participantes, ou pelos vários grupos de alunos. Os temas podem envolver brincadeiras, esportes, atividades industriais, econômicas e comerciais, prestação de serviços, meio ambiente e outros de interesse do grupo. As discussões proporcionadas nessa etapa podem levar ao desenvolvimento do raciocínio estatístico, mais especificamente o raciocínio sobre os dados, pois os alunos reconhecerão a necessidade dos dados, seus diferentes tipos, os quais produzem diferentes formas de recolhas e de tratamento. É nessa etapa que os alunos farão questionamentos do tipo: Como nossas questões iniciais serão respondidas? Como vamos coletar esses dados? Como deverão ser organizados de forma que nos ajudem a compreender o que está sendo investigado? 
Já em relação ao pensamento estatístico, nessa primeira etapa há uma maior evidência da primeira dimensão descrita por Wild e Pfannkuch (1999), o ciclo investigativo. É nesse momento que os alunos começam a visualizar todas as etapas de uma investigação estatística, pois escolher o tema requer definir o problema, planejar no sentido de identificar as variáveis envolvidas, discutir o plano de amostragem e como os dados deverão ser organizados, além compreender o que se busca explorar, elaborar hipóteses e generalizações. Ainda que não haja o domínio dos conteúdos estatísticos envolvidos em cada uma dessas ações, é nesse primeiro momento que ocorrem discussões no sentido de 0 aluno perceber a necessidade e a importância das ferramentas estatísticas. Entendemos que é nesse momento também que o professor deve intervir como mediador, a fim de encorajar os alunos a se envolverem numa atividade investigativa.

2) Pesquisa exploratória - Essa etapa da Modelagem se configura como importante para o desenvolvimento da experiência de campo, ajudando a gerar um comportamento mais atento, mais sensivel e mais crítico, atributos importantes na formação de uma postura investigativa. Assim, ao trabalhar um tema, procura-se identificar as várias dimensões ou aspectos que compõem a realidade. Por exemplo, ao se trabalhar o tema indústria cerâmica, explora-se aspectos que constituem essa realidade, sejam eles políticos, sociais, econômicos, estruturais, entre outros. Os dados coletados são de natureza qualitativa e quantitativa. Nessa segunda etapa pode-se desenvolver dois tipos importantes de raciocínio. Novamente o raciocínio sobre os dados, mas agora de maneira mais intensa de forma que o aluno, ao trabalhar nessa etapa, seja capaz de categorizar dados como quantitativos e qualitativos e suas subclassificações. Além desse tipo de raciocínio, tem-se, também, o raciocínio sobre as amostras, pois obter dados requer uma discussão sobre o plano de amostragem. Essa discussão é estendida entre alunos e alunos e professores, ou seja, é nessa etapa que a mediação do professor deve ser de tal forma que os alunos desenvolvam a capacidade de realizar amostragens que representem de forma satisfatória a população.

No que se refere ao pensamento estatístico percebe-se nessa etapa fortes relações com a segunda dimensão do pensamento estatístico, denominada por Wild e Pfannkuch (1999) de ciclo interrogativo, pois é nesse momento que se levanta questões do nível macro e micro da pesquisa. Conforme descrito por Burak (2019), é nessa etapa que procura-se identificar as várias dimensões ou aspectos que compõem a realidade, e fazer isso requer a geração de ideias, buscar informações, interpretar, criticar e julgar, que compõem a segunda dimensão do pensamento estatístico. Conforme dissemos, é nessa etapa que os alunos reconhecem a necessidade dos 
dados de forma mais categórica além de desenvolver planos mais elaborados de coleta de dados. Tal fato abarca uma outra dimensão do pensamento estatístico, denominada tipos fundamentais de pensamento estatístico - reconhecimento da necessidade dos dados, transnumeração, raciocinar com modelos estatísticos, interagir a estatística com o contexto. Essa dimensão está associada à consciência de que conclusões sobre um determinado contexto só podem ser tiradas com base em uma análise cuidadosa e sistemática.

3) Levantamento do problema - O levantamento do problema é a terceira etapa do método de Modelagem e é feito com base nos dados coletados na etapa da pesquisa exploratória. A ação investigativa, ao traduzir em dados quantitativos algumas observações, confere nova conotação aos dados numéricos obtidos, possibilitando a discussão e o estabelecimento de relações.

$\mathrm{Na}$ Modelagem Matemática, os problemas apresentam características distintas dos problemas apresentados pela maioria dos livros textos, pois são levantados a partir da coleta dos dados. Assim, problemas são elaborados com base nos dados coletados na pesquisa de campo; têm, geralmente, caráter genérico; estimulam a pesquisa e a organização dos dados; e favorecem a compreensão mais geral de uma determinada situação.

É na etapa do levantamento do problema que ocorre a transição entre linguagens diferentes; a primeira transição é da linguagem natural para a linguagem sistemática, que ocorre quando uma situação da realidade é transformada em informações; a segunda é a transição da linguagem sistemática para a linguagem matemática, que ocorre quando as informações são transformadas por meio de hipóteses simplificadas em um modelo matemático/estatístico. Entendemos que essa etapa permite potencializar o desenvolvimento dos raciocínios sobre dados, distribuição, centro e variabilidade. Há que se considerar que o professor tem um papel fundamental nessa etapa na qualidade de mediador, conduzindo e encorajando os alunos a trabalhar mais intensamente com esses tipos de raciocínio. Como é nessa etapa que ocorrerá a organização preliminar e exploração dos dados, além do planejamento mais detalhado das análises, ela é marcada por duas dimensões do pensamento estatístico: o ciclo investigativo, já que envolve o problema, as análises e conclusões; e os tipos de pensamento, os gerais e os fundamentais. Os gerais porque requerem a tomada de atitudes para a resolução de problemas cotidianos e os fundamentais porque inicia-se o exercício de transnumeração em seu primeiro estágio. 
4) Resolução do problema e o desenvolvimento do conteúdo matemático no contexto do tema - A quarta etapa da Modelagem trata da resolução do problema. Os problemas levantados determinam os conteúdos a trabalhar. Pautados pelo contexto do tema, podem ser desenvolvidos vários conteúdos matemáticos, estatísticos e não matemáticos provenientes dos dados coletados e das hipóteses levantadas pelo professor e/ou pelo grupo.

É nessa etapa da Modelagem Matemática que se faz uso de todas as ferramentas matemáticas/estatísticas disponíveis. Na resolução de um problema ou de uma situaçãoproblema, os conteúdos matemáticos ganham importância e significado. As operações, as propriedades e os diversos campos da Matemática que se destacam nessa fase, conferem significados aos conteúdos. Adicionalmente, questões não matemáticas oriundas dos dados podem merecer atenção ao envolver assuntos como meio ambiente, problemas sociais, políticos, culturais e econômicos, entre outros.

Às vezes, acontece que um conteúdo necessário para a resolução de um problema ainda não tenha sido apresentado ao aluno. É, então, um momento importante para que o professor, na condição de mediador, ofereça ao aluno a aquisição desse conhecimento.

Vê-se que essa etapa tem como objetivo a tradução do contexto por meio dos dados coletados. Para que isso ocorra é necessário a organização dos dados em tabelas e gráficos, a determinação das medidas de posição central e de dispersão, e estabelecimento de relações entre algumas variáveis envolvidas. Com isso, trata-se de uma etapa oportuna ao desenvolvimento dos seguintes tipos de raciocínio: sobre representação de dados, sobre medidas estatísticas e sobre associação. Esses tipos de raciocínio estão vinculados à terceira dimensão do pensamento estatístico, denominada tipos de pensamento, a qual é caracterizada pela adequação das ferramentas estatística ao contexto que está sendo investigado. Está associado a um exercício de transnumeração, que corresponde à exploração, análise e apresentação de números em formas de tabelas, gráficos e medidas, sejam elas de resumo ou de dispersão. É nessa etapa que ocorre também a consideração sobre a variação. Englobar isso tudo requer uma propensão para procurar significados mais profundos, sendo lógico, aberto e observador. Por isso, há que se estar envolvido com as ideias que estão associadas aos dados e ao contexto, e tais posicionamentos compõem a quarta dimensão do pensamento estatístico.

5) Análise crítica das soluções - Essa etapa da Modelagem é muito rica e especial para analisar e discutir as soluções encontradas. É um momento em que se fazem as considerações e 
avaliações das hipóteses construídas na fase de levantamento dos problemas. Possibilita tanto 0 aprofundamento de aspectos matemáticos como os aspectos não matemáticos envolvidos no tema. Sob o aspecto da Matemática, analisa-se a coerência e a consistência lógica das soluções encontradas. É nesse estágio que se discute os cuidados com a linguagem e com as restrições que se fazem necessárias em muitas ocasiões. Tão importante quanto trabalhar os aspectos matemáticos das situações, os não matemáticos, são essenciais para construção e consolidação de valores e de atitudes permanentes e essenciais para a formação do aluno.

Essa etapa pode ser entendida como o momento da validação das informações obtidas nas etapas 2 e 3 . Além de oportunizar um olhar cuidadoso para os tipos de raciocínio desenvolvidos em cada dessas fases, essa etapa também favorece o desenvolvimento do raciocínio sobre incerteza e inferência já que é possível inquirir o que pode ser inferido com base em uma amostra. Isso implica o momento de checar os pontos a favor e contra às conclusões que estão sendo realizadas, de questionar a confiabilidade das informações, utilidade das ideias e geração de novas ideias. Nessa etapa há que se raciocinar com os modelos estatísticos e interagir a Estatística com o contexto, mas, para isso, os alunos devem estar curiosos, abertos e atentos. Tais ações envolvem as quatro dimensões do pensamento estatístico, pois entendemos que é nessa etapa que o aluno é convidado a olhar de maneira mais sistemática para o processo de investigação estatística como um todo.

Com a finalidade de sistematizar a discussão feita nesse item, apresentamos resumidamente no Quadro 1, os tipos de raciocínio estatístico e elementos do pensamento estatístico que podem ser explorados em cada uma das etapas da Modelagem Matemática.

Quadro 1: Relações entre etapas da Modelagem Matemática, tipos de raciocínio estatístico e elementos do pensamento estatístico

\begin{tabular}{|c|c|c|}
\hline $\begin{array}{c}\text { Etapas da Modelagem } \\
\text { Matemática }\end{array}$ & Tipos de raciocínio & $\begin{array}{c}\text { Dimensão do pensamento } \\
\text { estatístico }\end{array}$ \\
\hline $1^{\text {a }}$ etapa - Escolha do tema & Raciocínio sobre os dados & Ciclo investigativo \\
\hline $\begin{array}{c}2^{\mathrm{a}} \text { etapa - Pesquisa } \\
\text { exploratória }\end{array}$ & $\begin{array}{l}\text { Raciocínio sobre os dados } \\
\text { Raciocínio sobre amostras }\end{array}$ & $\begin{array}{c}\text { Ciclo interrogativo } \\
\text { Tipos fundamentais do pensamento } \\
\text { estatístico }\end{array}$ \\
\hline $\begin{array}{c}3^{a} \text { etapa - Levantamento } \\
\text { dos problemas }\end{array}$ & $\begin{array}{l}\text { Raciocínio sobre representação de } \\
\text { dados } \\
\text { Raciocínio sobre medidas estatísticas } \\
\text { Raciocínio e sobre associação }\end{array}$ & $\begin{array}{c}\text { Ciclo investigativo } \\
\text { Tipos gerais e fundamentais do } \\
\text { pensamento estatístico }\end{array}$ \\
\hline
\end{tabular}




\begin{tabular}{|c|c|c|}
\hline $\begin{array}{c}4^{a} \text { etapa - Resolução do(s) } \\
\text { problema(s) e o } \\
\text { desenvolvimento do conteúdo } \\
\text { matemático no contexto do } \\
\text { tema }\end{array}$ & $\begin{array}{l}\text { Raciocínio sobre representação de } \\
\text { dados } \\
\text { Raciocínio sobre medidas estatísticas } \\
\text { Raciocínio e sobre associação }\end{array}$ & $\begin{array}{c}\text { Tipos fundamentais do pensamento } \\
\text { estatístico } \\
\text { Disposições }\end{array}$ \\
\hline Análise crítica das soluções & $\begin{array}{l}\text { Raciocínio sobre representação de } \\
\text { dados } \\
\text { Raciocínio sobre medidas estatísticas } \\
\text { Raciocínio e sobre associação } \\
\text { Raciocínio sobre incerteza }\end{array}$ & $\begin{array}{c}\text { Ciclo investigativo } \\
\text { Ciclo interrogativo } \\
4 \text { dimensões do pensamento } \\
\text { Disposição }\end{array}$ \\
\hline
\end{tabular}

Fonte: Elaboração dos Autores

Note-se que os tipos de raciocínio e os elementos do pensamento estatístico não pertencem exclusivamente à uma única etapa da atividade de Modelagem Matemática apresentada no Quadro 1, nós apenas destacamos o que nos pareceu ser mais marcante em cada uma dessas etapas. O processo investigativo é dinâmico, e, por essa razão, é marcado pela retomada das etapas já vivenciadas, com um olhar para o que ainda há que ser feito.

Além disso, compreendemos que essas relações não precisam ser rigidamente seguidas, pois representam apenas uma tentativa de organizar o processo de modelagem matemática quando utilizado com vistas a favorecer o ensino e a aprendizagem de Estatística. Com base no Quadro 1 também é possível perceber que a Modelagem Matemática apresenta as diferentes fases de uma pesquisa estatística - abordagem de uma situação-problema, decisão sobre a melhor forma da coleta de dados, coleta e análise de dados reais, formulação e conclusões sobre o problema proposto - por essa razão, compreendemos o motivo pelo qual essa abordagem tem recebido destaque nas pesquisas em Educação Estatística, conforme apontado por Santos (2015).

Além do mencionado, destacamos que a Modelagem Matemática possui considerável relevância quando se planeja os processos de ensino e de aprendizagem de Estatística com vistas ao desenvolvimento das competências raciocínio e pensamento, uma vez que a capacidade de aplicar o conhecimento estatístico geralmente é muito mais difícil do que se supõe, porque requer não apenas conhecimento técnico - como preparar um gráfico ou calcular uma média -, mas também conhecimento estratégico - saber quando usar um conceito, um gráfico, um dado. Problemas e exercícios em livros didáticos tendem a se concentrar apenas no conhecimento técnico, enquanto o ambiente de modelagem matemática inclui também o conhecimento estratégico. 


\section{Considerações}

Neste artigo evidenciamos que as etapas sugeridas no processo de Modelagem Matemática conforme proposto por Burak (2019) possuem relações significativas com os tipos de raciocínio estatístico e os elementos do pensamento estatístico, o que nos permite afirmar que 0 processo de investigação estatística é o próprio ambiente de Modelagem Matemática, ou seja, esse ambiente pode favorecer 0 desenvolvimento dessas competências.

Esse ambiente proporciona que o aluno vivencie os desafios que permeiam um processo de investigação estatística, o que é necessário para que as pessoas compreendam as particularidades dessa área do conhecimento. Ele também permite dar sentido aos vários objetos estatísticos e envolve os alunos em um ciclo de investigação no qual formas de raciocínio são necessárias.

Com mediação do professor nas fases iniciais da Modelagem Matemática, os alunos devem identificar quais são os dados necessários para responder seus questionamentos iniciais e como esses devem ser coletados. Essa tarefa possibilita discussões sobre tipos de variáveis, formas de coleta de dados, sejam através do censo ou amostra, como também estudos acerca das técnicas de amostragem.

Nas fases subsequentes, após a coleta de dados, tópicos da estatística descritiva, como gráficos, tabelas, medidas de posição central e de dispersão, correlação e regressão linear, podem ser estudadas de forma a colaborar com a organização, análise e interpretação dos resultados. Já nas fases finais da Modelagem Matemática, de posse das relações verificadas no processo investigativo, estas são analisadas e comparadas com as hipóteses estabelecidas. Essas análises devem possibilitar tirar conclusões e fazer previsões para a população, momento em que os tópicos de inferência podem ser explorados.

Ademais, este estudo apontou os diferentes tipos de raciocínio e dimensões do pensamento estatístico que podem ser explorados em cada uma das etapas da Modelagem Matemática. Cabe ressaltar que, conforme apontado em nossa fundamentação teórica, existem outras concepções de Modelagem Matemática e as relações aqui consideradas são válidas para a concepção adotada neste estudo. Diferentes concepções podem trazer convergências com outras competências da Educação Estatística, a literacia, por exemplo. 


\section{Referências}

ALMOULOUD, Saddo Ag; SILVA, Maria José Ferreira da. Construção do referencial teórico de uma pesquisa educacional. In OLIVEIRA, Gerson Pastre de. (Org.), Pesquisa em Educação Matemática: um olhar sobre a metodologia. Curitiba: CRV, 2019, p. 49-82.

BARBOSA. Jonei Cerqueira. Modelagem na Educação Matemática: contribuições para o debate teórico. REUNIÃO ANUAL DA ASSOCIAÇÃO NACIONAL DE PÓS-GRADUAÇÃO E PESQUISA EM EDUCAÇÃO, 24, 2001, Caxambu. Anais da XXIV Reunião Anual da ANPEd. Rio de Janeiro: ANPEd, 2001, p. 1-15.

BASSANEZI, Rodney Carlos. Ensino-aprendizagem com modelagem matemática: uma nova estratégia. São Paulo: Contexto, 2011.

BEN-ZVI, Dani; ARIDOR-BERGER, Keren. Children's wonder how to Wander between data and context. In: BEN-ZVI, Dani; MAKAR, Katie (Ed.). The teaching and learning of Statistics: international perspectives. New York: Springer, 2016, p. 25-37.

BIEHLER, Rolf; FRISCHEMEIER, Daniel; READING, Chris; SHAUGHNESSY, Michael. Reasoning about data. In: BEN-ZVI, Dani; MAKAR, Katie; GARFIEL, Joan. (Org.) International Handbook of Research in Statistics Education. Gewerbestrasse: Springer International, 2018, p. 138-186.

BIEMBENGUT, Maria Salett; HEIN, Nelson. Modelagem matemática no ensino. São Paulo: Contexto, 2013.

BRASIL. Ministério da Educação. Secretaria de Educação Básica. Base Nacional Comum Curricular. Ensino Médio. Brasília: MEC/SEB, 2018.

BURAK, Dionísio. A Modelagem Matemática na perspectiva da Educação Matemática: olhares múltiplos e complexos. Educação Matemática sem Fronteiras, Chapecó, v. 1, n. 1, p. 96-111, jan./jun. 2019.

BURAK, Dionísio. Modelagem Matemática e a sala de aula. In: I ENCONTRO PARANAENSE DE MODELAGEM EM EDUCAÇÃO MATEMÁTICA, 2004, Londrina. Anais do I EPREM: Modelagem Matemática: práticas, críticas e perspectivas de Modelagem na Educação Matemática. Londrina: UEL, 2004, 208-222.

BURAK, Dionísio. Modelagem matemática: ações e interações no processo de ensino aprendizagem. 1992. 460f. Tese (Doutorado em Educação) — Faculdade de Educação. Universidade Estadual de Campinas. Campinas.

CAMPOS, Celso Ribeiro. A Educação Estatística: uma investigação acerca dos aspectos relevantes à didática da Estatística em cursos de graduação. 2007. 256f. Tese (Doutorado em Educação Matemática) - Instituto de Geociências e Ciências Exatas. Universidade Estadual Paulista. Rio Claro.

CAMPOS, Celso Ribeiro; WODEWOTZKI, Maria Lúcia Lorenzetti; JACOBINI, Otávio Roberto. Educação Estatística: teoria e prática em ambientes de modelagem matemática. Belo Horizonte: Autêntica, 2011. 
CAZORLA, Irene Maurício; SILVA JÚNIOR, Antonio Vital; SANTANA, Eurivalda Ribeiro dos Santos. Reflexões sobre o ensino de variáveis conceituais na educação básica. REnCiMa, São Paulo, v. 9, n. 2, p. 354-373, 2018.

COUTINHO, Cileda de Queiroz e Silva; CAMPOS, Celso Ribeiro. Metodologia quantitativa e mista. In: OLIVEIRA, Gerson Pastre de (Org.). Pesquisa em Educação Matemática: um olhar sobre a metodologia. Curitiba: CRV, 2019, p. 83-108.

D'AMBROSIO, Ubiratan. Da realidade à ação: reflexos sobre Educação e Matemática. São Paulo: Summus, 1986.

EICHLER, Andreas. Statistics teachers and classroom practices. In: BATANERO, Carmen; BURRILL, Gail; READING, Chris. Teaching statistics in school mathematics-challenges for teaching and teacher education. New York: Springer, 2011. p. 175-186.

EVANGELISTA, Dilson Henrique Ramos; EVANGELISTA, Cristiane Johann; SANTOS, Roziane Sobreira. $O$ ensino e aprendizagem de Estatística com uso de projetos de modelagem e tecnologias digitais. Brazilian Journal of Development, Curitiba, v. 6, n. 1, p. 4181-4191, jan. 2020.

FIORENTINI, Dario; LORENZATO, Sérgio. Investigação em Educação Matemática: percursos teóricos e metodológicos. Campinas: Autores Associados, 2006.

MACHADO, Minéia Bortole. Modelagem matemática como ambiente de aprendizagem de Estatística na educação Básica. 2017. 155f. Dissertação (Mestrado em Ensino de Matemática) Instituto de Matemática. Universidade Federal do Rio Grande do Sul. Porto Alegre.

MEYER, João Frederico da Costa; CALDEIRA, Ademir Donizetti; MALHEIROS, Ana Paula dos Santos. Modelagem em Educação Matemática. Belo Horizonte: Autêntica Editora, 2011.

MOORE, Thomas. Teaching statistics: resources for undergraduate instructors. Washington: F. and S. Gordon, 2001.

PERIN, Andréa Pavan. Educação Estatística Crítica: um estudo das práticas discentes em um curso de tecnologia. 2019. 267f. Tese (Doutorado em Educação Matemática) - Instituto de Geociências e Ciências Exatas. Universidade Estadual Paulista. Rio Claro.

PFANNKUCH, Maxine; RUBICK, Amanda. An exploration of students' statistical thinking with given data. Statistics Education Research Journal, v.1, n. 2, p. 4-21, apr. 2002

PFANNKUCH, Maxine. Reimagining curriculum approaches. In: BEN-ZVI, Dani; MAKAR, Katie; GARFIEL, Joan. (Ed.). International Handbook of Research in Statistics Education. Gewerbestrasse: Springer International, 2018, p. 384-406.

PFANNKUCH, Maxine; WILD, Chris. Statistical thinking: how can we develop it? In: INTERNATIONAL STATISTICAL INSTITUTE CONFERENCE, 54, 2018. Proceedings of the $54^{\text {th }}$ ISIC. Voorburg: International Statistical Institute, 2003, p. 567-574.

PRATT, Dave; KAZAK, Sibel. Research on uncertainty. In: BEN-ZVI, Dani; MAKAR, Katie; GARFIEL, Joan. (Ed.) International Handbook of Research in Statistics Education. Gewerbestrasse: Springer International, 2018, p. 193-228. 
SAMÁ, Suzi. Caminhos trilhados pelo GT12 nas pesquisas em Educação Estatística no Brasil. In: SEMINÁRIO INTERNACIONAL DE PESQUISA EM EDUCAÇÃO MATEMÁTICA, 7, 2018, Foz do Iguaçu. Annais do VII SIPEM. Foz do Iguaçu: SBEM, 2018, p. 1-12.

SANTOS, Rodrigo Medeiros. Estado da arte e história da pesquisa em Educação Estatística em programas brasileiros de pós-graduação. 2015. 348f. Tese (Doutorado em Educação) Faculdade de Educação. Universidade Estadual de Campinas. Campinas.

SNEE, Ronald. Discussion: development and use of statistical thinking: a new era. International Statistical Review, v. 67, n. 3, p. 255-258, dec. 1999.

SNEE, Ronald. Statistical thinking and its contribution to total quality. The American Statistician, Califórnia, v. 44, n. 2, p. 116-121, 1990.

SOARES, José Aílton Rodrigues. Modelagem matemática como estratégia de ensino de tópicos de Estatística na formação básica técnica. 2017. 172f. Dissertação (Mestrado em Matemática) Universidade Federal do Tocantins. Arraias.

TRAINOTTI, Andressa. A Educação Estatística e a modelagem matemática na formação crítica dos estudantes do Ensino Médio de escolas do município de Rio do Sul - SC. 2019. 95f. Dissertação (Mestrado em Ensino de Matemática) - Instituto de Matemática. Universidade Federal do Rio Grande do Sul. Porto Alegre.

WILD, Chris; PFANNKUCH, Maxine. Statistical thinking in emperical enquiry. Internacional Statistics Review, New York, v. 67, n. 4, p. 223-265, 1999.

ZIEFFLER, Andrew; GARFIELD, Joan; FRY, Elizabeth. What is Statistics Education? In: BEN-ZVI, Dani; MAKAR, Katie; GARFIEL, Joan. (Ed.). International Handbook of Research in Statistics Education. Gewerbestrasse: Springer International, 2018, p. 37-71. 